\title{
Optimal allocation of multi-type FACTS devices in power systems based on power flow entropy
}

\author{
Canbing LI ( $ه)$, Liwu XIAO, Yijia CAO, \\ Qianlong ZHU, Baling FANG, Yi TAN,
} Long ZENG

\begin{abstract}
Flexible AC transmission systems (FACTS) devices can effectively optimize the distribution of power flow. Power flow entropy can be applied as a measure of load distribution. In this paper, a method is proposed to optimize the distribution of power flow with the coordination of multi-type FACTS devices and establishes the corresponding mathematical models. The modified group searcher optimization (GSO) algorithm is proposed, in which the angle search is combined with chaotic search model to avoid jumping into local optimization. Compared with the different optimal allocation of multi-FACTS devices, the optimal allocation of multi-FACTS devices is achieved under the economic constraints. The locations obtained by this method can achieve the purpose of balancing power flow and enhancing the system performances. The simulations are demonstrated in an IEEE 118-bus power system with two classical types of FACTS, namely static var compensator (SVC) and thyristor controlled series Compensator (TCSC). The simulation results show that the proposed method is feasible and effective.
\end{abstract}

Keywords Chaotic search model, Flexible AC transmission system (FACTS), Group searcher optimization (GSO), Power flow entropy

CrossCheck date: 22 May 2014

Received: 10 July 2013/ Accepted: 15 May 2014/Published online: 5 June 2014

(C) The Author(s) 2014. This article is published with open access at Springerlink.com

C. LI, L. XIAO, Y. CAO, Q. ZHU, B. FANG, Y. TAN, L. ZENG,

College of Electrical and Information Engineering, Hunan

University, Changsha 410082, China

$(\triangle)$ e-mail: licanbing@gmail.com

\section{Introduction}

Flexible AC transmission systems (FACTS) devices can make power system more controllable and safe [1]. They can improve the stability of the system and the power transmission capacity and improve the distribution of power flow and reduce the transmission loss through changing the parameters of power transmission system [2-4].

Presently, based on different objective functions, there are many researches on allocation and operations of FACTS with different algorithms, such as genetic algorithm (GA), particle swarm optimization (PSO) or bacterial swarming algorithm (BSA) [5]. Reference [6] studied on minimum singular value index and sensitivity of FACTS controllers to select the optimized location to improve the power transmission capacity. Respectively, the objective functions of minimization of real power loss in transmission lines and voltage deviation at load buses were proposed to identify parameters and locations of FACTS [7, 8]. Considering the vulnerability and network security indices, reference [9] presented a method to improve network security margin by optimizing locations of FACTS devices. In order to eliminate or alleviate the line overloads, reference [10] presented the method based on the contingency severity index (CSI) described by a real power flow performance index (PI) to determine placement of multi-FACTS devices, and the optimized parameters of FACTS devices could be obtained using GA. Considering the capability characteristics of the SVC and TCPAR, reference [11] analyzed their impacts on composite power system reliability by using evaluation method (EM). To minimize the real power losses and improve voltage profile, a novel bacterial swarming algorithm (BSA) was proposed to select the optimal locations and control parameters of multi-type FACTS devices [12]. Considering different scenarios and using harmony search algorithm (HSA) and GA for placement of multi-FACTS 


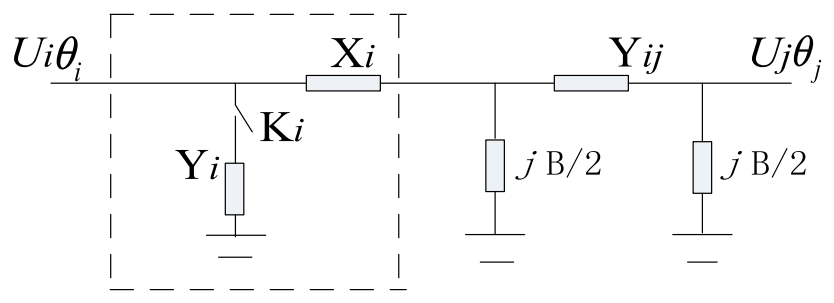

Fig. 1 FACTS model

devices, reference [13] verified that FACTS devices could improve the power system stability margins, maximum voltage stability margin and reduce losses in the network. The objective functions of maximum system load ability of power system and minimum investment cost were achieved by using GA and PSO, which solved the optimal location and parameter settings of multiple TCSCs problem [14]. Respectively, the effects of FACTS on improving the system load ability and enhancing the TTC value by using GA and evolutionary programming (EP) had been discussed $[15,16]$. However, among the above documents, there are few researches related to the aspect of the distribution of power flow.

Based on the entropy theory, a novel concept of power flow entropy has been proposed to measure the distribution of power flow [17]. The smaller the value of power flow entropy is, the more orderly and equal the distribution of power flow will be. The previous studies indicate that homogeneous distribution of power flow, which can improve the security of the system, helps to reduce the probability of cascading failures and large blackouts due to chain reaction in power grids [17-21].

In this paper, the proposed method is for the coordination of multi-type FACTS devices based on power flow entropy. Through the modified GSO algorithm, the optimal locations and control parameters of multi-type FACTS devices are selected and yield efficiency in equalization of distribution of power flow. The proposed method is applied in an IEEE 118-bus power system.

\section{FACTS' model and objective function}

\subsection{The steady state models of FACTS devices}

FACTS devices can be broadly classified into three types, namely shunt, series, and composite series and shunt. When FACTS devices are installed in the transmission system, the model of FACTS devices can be unified as shown in Fig. 1 [22]. Intuitively, FACTS devices affect the system flow distribution mainly through three key parameters, the bus voltage, the line impedance, and the beginning and the end of the relative phase angle [23]. $\mathrm{K}_{i}$ represents a

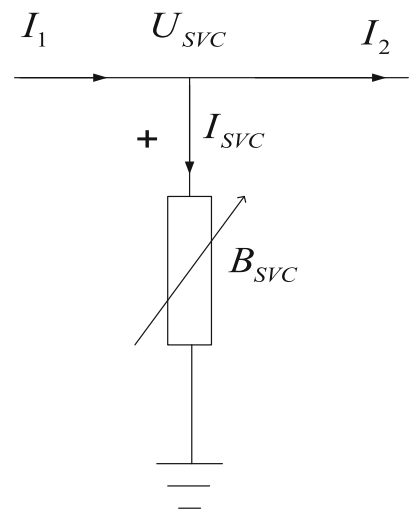

Fig. 2 Equivalent steady-state circuit of SVC

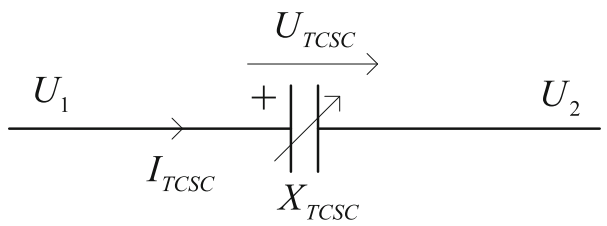

Fig. 3 Equivalent steady-state circuit of TCSC

switch here. When the switch $\mathrm{K}_{i}$ is closed, it represents the shunt or composite series and shunt type; when the switch $\mathrm{K}_{i}$ is opened, it represents the series type.

In this paper, two classical types of FACTS, SVC and TCSC, are chosen. SVC enhances the power transfer capability of the line by improving the voltage of node, which is paralleled in the system as a variable susceptance as shown in Fig. 2. And TCSC directly involves in modifying the reactance of the line as a capacitive or inductive compensation to improve the power transfer capability of the line as shown in Fig. 3.

When SVC and TCSC are incorporated and installed into power system, the node admittance matrix of the system is:

$Y^{\prime}=Y+\left[\begin{array}{ccccc}0 & 0 & \cdots & 0 & 0 \\ 0 & \Delta y_{i j} & \cdots & -\Delta y_{i j} & 0 \\ \vdots & \vdots & \vdots & \vdots & \vdots \\ 0 & -\Delta y_{i j} & \cdots & \Delta y_{i j} & 0 \\ 0 & 0 & \cdots & 0 & 0\end{array}\right]$

where $Y$ and $Y^{\prime}$ represent the node admittance matrix of the system before and after the installation of FACTS respectively; $i$ and $j$ represent the nodes installed FACTS devices respectively. In the power flow equations, Jacobian matrix does not change in size. Therefore, in this paper, it only needs to modify the corresponding node admittance of the nodes and branches that have installed FACTS devices, which is conducive to this study. 


\subsection{Objective function}

The theory of entropy is applied to law of thermodynamics firstly. Then it is applied to information science, and statistical physics, etc. Entropy is a measure of the chaos and disorder of the system. A complex system may be in different states, which are represented by $\left\{X_{1}, \ldots\right.$, $\left.\mathrm{X}_{m}\right\} . P\left(X_{i}\right)$ represents the probability that the system is in state $\mathrm{X}_{i}, i=1,2, \ldots, m$. Then the entropy of the system can be defined as:

$H=-C \sum_{k=1}^{m} P\left(X_{i}\right) \ln P\left(X_{i}\right)$

where $C$ is a constant, $m$ is the number of states.

Here the load rate of line $i$ can be expressed as

$\alpha_{i}=P_{i} / P_{i}^{\max }$

where $P_{i}$ represents the active power flow of line $i$; $P_{i}^{\max }$ represents the maximum transmission capacity of line $i$; and $i$ represents the number of transmission lines in the power grid.

The load rate of each line is not identical, and $\alpha_{i}$ belongs to range 0 to 1 . Therefore, the load rates of lines can be grouped into $M$ successive intervals, which are defined as $[0, u),[u, 2 u), \ldots,[(M-1) u, M \cdot u]$; the load rates of lines can be probabilistic in the interval $[(k-1) u, k u]$, as follows:

$P(k)=\frac{l_{k}}{N}$

where $P(k)$ represents the proportion of the total lines in the $k^{\text {th }}$ interval; $l_{k}$ represents the number of lines in the $k^{\text {th }}$ interval group of conditions; and $N$ represents the total number of lines.

Power flow in power grid is bidirectional, thus the entropy value is different in the two directions. In this paper, one of them is chosen as the research direction.

The power flow entropy $H$ is defined as

$H=C \sum_{k=1}^{M} P(k) \ln P(k)$

where $M$ represents the total group number of the load rates, here $M=100$; and $C$ is a constant, here $C=-\ln 10$.

In this paper, the optimal placements of multi-FACTS devices are selected to make the value of power flow entropy $H$ minimum and achieve the balance of power flow. So the objective function is as

$\min H=H\left(X_{n}, B_{n}\right)$

where $X_{n}=\left[x_{1}, x_{2}, \ldots, x_{n}\right]$ and $B_{n}=\left[b_{1}, b_{2}, \ldots, b_{n}\right]$ represent the vector of reactance of TCSC and the vector susceptance of SVC respectively; and $n$ represents the installation number of FACTS devices.
Equation (5) shows that the value of power flow entropy $H$ provides the measure of the load distribution. The maximal value of power flow entropy is $C \cdot \log (1 / M)$, when all the states of the system are with the same probability $P(k)=1 / M$. It means that the system is the most disorderly. The minimal value is zero, when the load rate of each line is identical, namely there is no difference and the probability is 1 . It means that the system is the most orderly. Here, the value of power flow entropy $H$ is controlled by the reactance of TCSC and the susceptance of SVC.

\subsection{System constraints}

\subsubsection{Equality constraints}

Equality constraints of the node power balance equation are introduced into FACTS devices.

$P_{G i}-P_{L i}-V_{i} \sum_{j=1}^{N} V_{j}\left(G_{i j} \cos \theta_{i j}+B_{i j} \sin \theta_{i j}\right)=0$

$Q_{G i}-Q_{L i}-V_{i} \sum_{j=1}^{N} V_{j}\left(G_{i j} \sin \theta_{i j}-B_{i j} \cos \theta_{i j}\right)=0$

where $P_{G i}, Q_{G i}, P_{L i}, Q_{L i}$ represent active and reactive power generation at node $i$, active and reactive power flow at node $i$, respectively; $V_{i}$ and $V_{j}$ represent bus voltage magnitudes at nodes $i$ and $j$, respectively; $\theta_{i j}$ represents voltage angle between nodes $i$ and $j ; G_{i j}$ and $B_{i j}$ represent conductance and susceptance of the line with FACTS; and $N$ represents the number of nodes.

\subsubsection{Inequality constraints}

Generation limits:

$P_{G i}^{\min } \leq P_{G i} \leq P_{G i}^{\max }$

$Q_{G i}^{\min } \leq Q_{G i} \leq Q_{G i}^{\max }$

where $P_{G i}$ represents the active power generation with lower and upper limits represented by $P_{G i}^{\min }$ and $P_{G i}^{\max }$, and $Q_{G i}$ represents the reactive power generation with lower and upper limits represented by $Q_{G i}^{\min }$ and $Q_{G i}^{\max }$ at node $i$.

Power line limits:

$P_{L i}^{\min } \leq P_{L i} \leq P_{L i}^{\max }$

$Q_{L i}^{\min } \leq Q_{L i} \leq Q_{L i}^{\max }$

where $P_{L i}$ represents the active power flow of line $i$ with lower and upper limits represented by $P_{L i}^{\min }$ and $P_{L i}^{\max }$, and $Q_{L i}$ represents the active power flow of line $i$ with lower and upper limits represented by $Q_{L i}^{\min }$ and $Q_{L i}^{\max }$ at node $i$.

Voltages and voltage angles limits: 
$V_{i}^{\min } \leq V_{i} \leq V_{i}^{\max }$

$\theta_{i}^{\min } \leq \theta_{i} \leq \theta_{i}^{\max }$

where $V_{i}$ represents voltage magnitude with lower and upper limits represented by $V_{i}^{\min }$ and $V_{i}^{\max }$, and $\theta_{i}$ represents voltage angle with lower and upper limits represented by $\theta_{i}^{\min }$ and $\theta_{i}^{\max }$ at node $i$.

FACTS limit:

$x_{i}^{\min } \leq x_{i} \leq x_{i}^{\max }$

$b_{i}^{\min } \leq b_{i} \leq b_{i}^{\max }$

where $x_{i}$ represents the compensated reactance of the line by TCSC with lower and upper limits represented by $x_{i}^{\min }$ and $x_{i}^{\max }$, and $b_{i}$ represents the compensated susceptance of the line by SVC with lower and upper limits represented by $b_{i}^{\min }$ and $b_{i}^{\max }$.

\subsection{Economic constraints}

Because FACTS devices are costly, the number of installed FACTS devices is limited. The total cost of the investment $C$ includes the investment costs of TCSC and SVC [24, 25].

$C=\sum_{k=1}^{p} c_{T C S C} \cdot x_{T C S C}(k) \cdot i_{l k}^{2}+\sum_{k=1}^{q} c_{S V C} \cdot\left|b_{S V C}\right|(k) \cdot v_{k}^{2}$

where $p$ and $q$ represent the number of TCSC and SVC respectively; $c_{T C S C}$ and $c_{S V C}$ represent the investment cost per kvar-installed of TCSC and SVC respectively [26, 27]; $x_{T C S C}$ represents the series reactance by TCSC; $b_{S V C}$ represents the susceptance by SVC; $i_{l k}$ represents the active power flow through the transmission line; and $v_{k}$ represents the voltage magnitude at node $i$.

\section{Modified GSO algorithm and its application}

Static stability analysis of large complex power systems have many cases. The calculation of addressing arbitration based on traversing method is extremely time-consuming. In this paper, the modified GSO algorithm is adopted for installation location optimization, which can shorten the time of the simulation and obtain the best position.

Table 1 Investment cost per kVar-installed of FACTS

\begin{tabular}{ll}
\hline Type of FACTS & Investment cost $(\$ / \mathrm{kVar})$ \\
\hline SVC & 40 \\
TCSC & 50 \\
\hline
\end{tabular}

\subsection{Angle selection of GSO algorithm}

GSO algorithm search space size is determined by the maximum pursuit angle $\theta_{\max }$ and maximum pursuit distance $l_{\text {max }}$. Angle search is a key factor in the optimization process. In the standard GSO algorithm, it is presented by the following two formulas changing search [28-30].

$\varphi^{k+1}=\varphi^{k} \pm r \theta_{\max } / 2$

$\varphi^{k+1}=\varphi^{k}+r \alpha_{\max }$

where $r \in R^{n-1}$ is a uniformly distributed random sequence in the range $(0,1), \theta_{\max } \in R^{1}$ represents maximum pursuit angle, $\alpha_{\max } \in R^{1}$ is the maximum turning angle. Equations (18) and (19) represent the new randomly generated angles.

Accordingly, the change of angle search is randomly determined by the size of $r$. Thus, the angle search may be repeated and lead to the local optimum. To improve the process of angle search and avoid jumping into the local angle search, modifying angle change can make it have a certain purpose. Through changing the angle every time, chaos search is introduced (Tables 1, 2, 3).

\subsection{Chaos search algorithm model}

Chaos search algorithm has three important dynamic properties: stochastic property, regularity and ergodicity. In order to achieve the change of angle search process, Logistic map is introduced into angle search of GSO algorithm.

This map is defined by [31].

$r_{n+1}=\gamma r_{n}\left(1-r_{n}\right)$

where $n$ is the serial number of chaotic variables, and $\gamma$ is a chaotic attractor. When $\gamma=4$, system enters into a chaos state.

Using $r_{n}$ instead of $r$ in the original algorithm, the equations are:

$\varphi^{k+1}=\varphi^{k} \pm \gamma r_{n}\left(1-r_{n}\right) \theta_{\max } / 2$

$\varphi^{k+1}=\varphi^{k}+\gamma r_{n}\left(1-r_{n}\right) \alpha_{\max }$

Angle search process is random and ergodic, as a result, it avoids falling into local search and local optimum.

As shown in Fig. 4, optimization result using the modified GSO algorithm is better than that using the original algorithm.

The specific process are:

Step 1: Load the original data of system and FACTS;

Step 2: Generate the initial population of $n$ randomly, and the chaos of the original variables $r_{0}$; 
Step 3: Power flow calculation and data processing. Calculate the objective function value, and select a minimum of objective function as producer in the initial populations. $80 \%$ of the members of the initial population are looked as scroungers, and the rest of the members are looked as rangers;

Step 4: Update the placements of producer, scrounger and ranger, and calculate the objective function value correspondingly using the modified GSO algorithm;

Step 5: If the objective function value is smaller than the objective function value of the initial producer, the new producer replaces the original producer, and the initial producer is merged with scrounger or ranger, continuing to update;

Step 6: Judge the termination condition. If the termination condition (maximum number of iterations) is satisfied, the algorithm is terminated; if not, repeat Step 3 and Step 5;

Step 7: Terminate the algorithm, and output the final optimal producer.

\section{Case study}

To verify the proposed method, the IEEE 118-bus test system is taken into account to select the locations and parameters of multi-FACTS in this paper. This network consists of 54 generator buses and 186 branches [32, 33]. In the modified GSO algorithm, there are 50 initial populations, and the maximum number of iterations is 100 .

For the purpose of objective optimization, the number of FACTS, line load rate, line flow, and the loss of network are compared. The value of power flow entropy is 9.5208 as the initial accumulator value before installation of FACTS. In this paper, TCSC and SVC were tested respectively.

As shown in Fig. 5, when 15 TCSCs are installed, the value of power flow entropy is more than 9.1875 ; when 12 SVCs are installed, the value of power flow entropy is more than 9.3740. The comparison clearly shows that the regulatory capacity of TCSC for power flow is better than the regulatory capacity of SVC. However, considering the economic aspect, TCSC should be installed into the system in coordination with SVC to achieve optimal value. Through testing, when 4 SVCs and 6 TCSCs are chosen, the value of power flow entropy is 9.1956 .

Table 4 and Table 5 show that the coordination of multi-FACTS based on power flow entropy has obvious effects on the power system.

The corresponding load rates and active power of lines changed at the objective function in Table 4 . In fact, the changes of all load rates of lines before and after installation of FACTS devices are shown in Fig. 6. The section above zero level shows that load rates of lines are in positive growth, indicating that load rates of lines increased. The section below zero level shows that load

Table 2 Investment of installation

\begin{tabular}{lc}
\hline Types of FACTS & Total investment $(\$)$ \\
\hline 12 SVC & $304.9 \times 10^{4}$ \\
15 TCSC & $376.9 \times 10^{4}$ \\
4 SVC+6 TCSC & $331.69 \times 10^{4}$ \\
\hline
\end{tabular}

Table 3 Locations, the reactance and the susceptance of FACTS

\begin{tabular}{lll}
\hline Types of FACTS & Locations & $x_{t c s c}, b_{s v c}$ (p.u.) \\
\hline SVC1 & 9 & $(0,-1)$ \\
SVC2 & 29 & $(0,-0.30356)$ \\
SVC3 & 84 & $(0,-0.53839)$ \\
SVC4 & 102 & $(0,-0.78389)$ \\
TCSC5 & $30-26$ & $(0.04622,0)$ \\
TCSC6 & $36-34$ & $(0.01441,0)$ \\
TCSC7 & $66-62$ & $(0.11717,0)$ \\
TCSC8 & $72-24$ & $(0.10535,0)$ \\
TCSC9 & $77-76$ & $(0.07955,0)$ \\
TCSC10 & $80-77$ & $(0.02607,0)$ \\
\hline
\end{tabular}

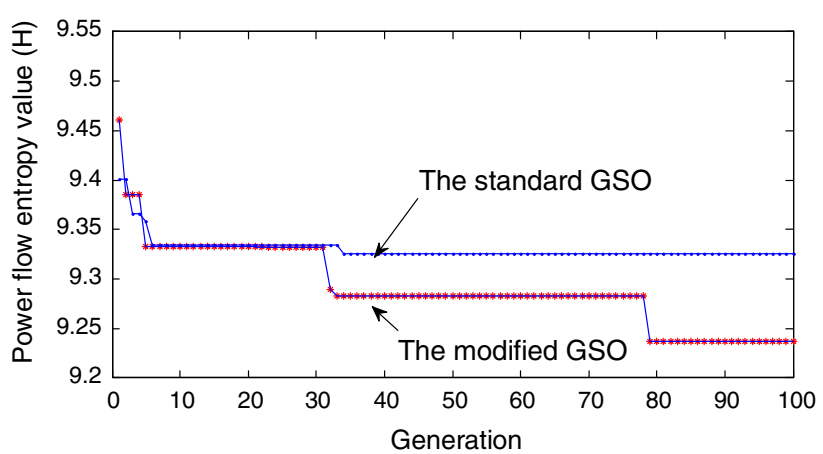

Fig. 4 Comparison of performance index evolution for GSO

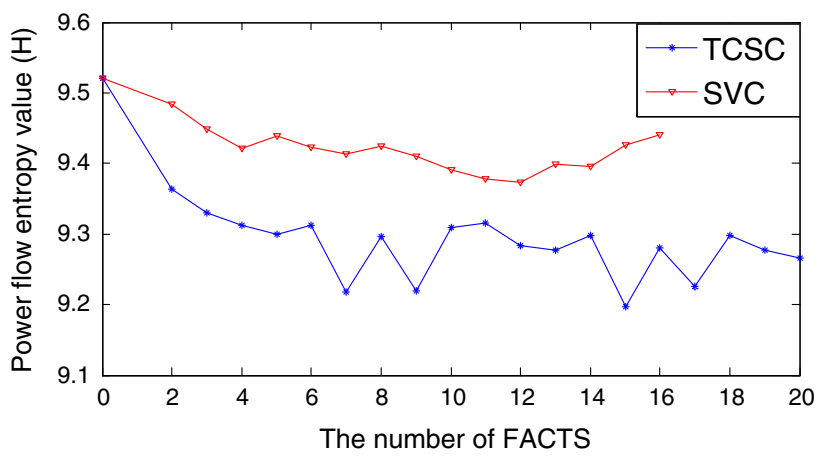

Fig. 5 Curve of entropy value $H$ with the number of FACTS 
Table 4 Change of load rate and power flow with and without FACTS

\begin{tabular}{llrllr}
\hline \multirow{2}{*}{ Number of branches } & \multicolumn{2}{l}{ Without FACTS } & & \multicolumn{2}{c}{ With FACTS } \\
\cline { 2 - 3 } & $\alpha_{l}$ (p.u.) & $\mathrm{P}_{l}$ (p.u.) & & $\alpha_{l}$ (p.u.) & $\mathrm{P}_{l}$ (p.u.) \\
\hline 21 & 0.83 & 1.2327 & & 0.721 & 1.0709 \\
39 & 0.435 & -0.0365 & & 0.3142 & -0.2638 \\
41 & 0.8962 & -1.0934 & & 0.6767 & -0.8256 \\
60 & 0.0169 & 0.0023 & & 0.2143 & -0.0293 \\
96 & 0.8604 & 1.9267 & & 0.7774 & 1.7408 \\
\hline
\end{tabular}

Table 5 Comparisons of system power generation and loss with and without FACTS

\begin{tabular}{lllll}
\hline FACTS & $P$ (p.u.) & $Q$ (p.u.) & $P_{\text {loss }}$ (p.u.) & $Q_{\text {loss }}$ (p.u.) \\
\hline Without FACTS & 38.0348 & 8.1993 & 1.3548 & -5.3372 \\
With FACTS & 37.9637 & 9.7655 & 1.2837 & -3.7934 \\
\hline
\end{tabular}

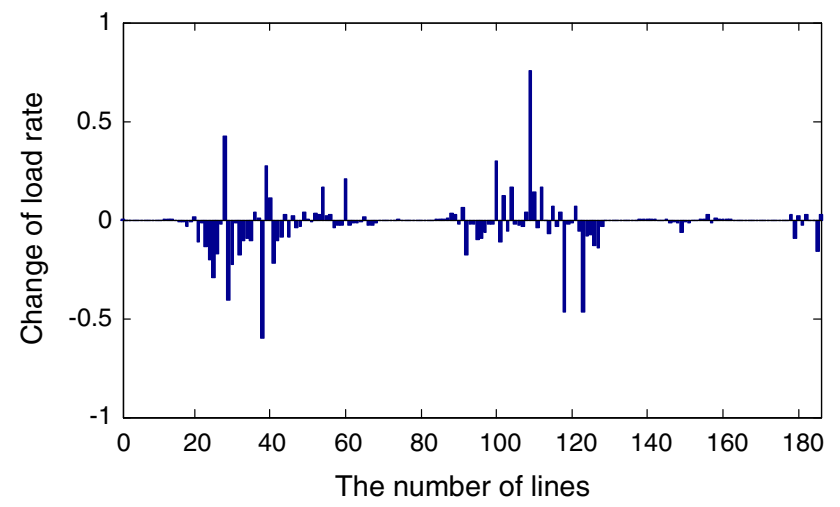

Fig. 6 Load rates changes before and after installation of FACTS devices

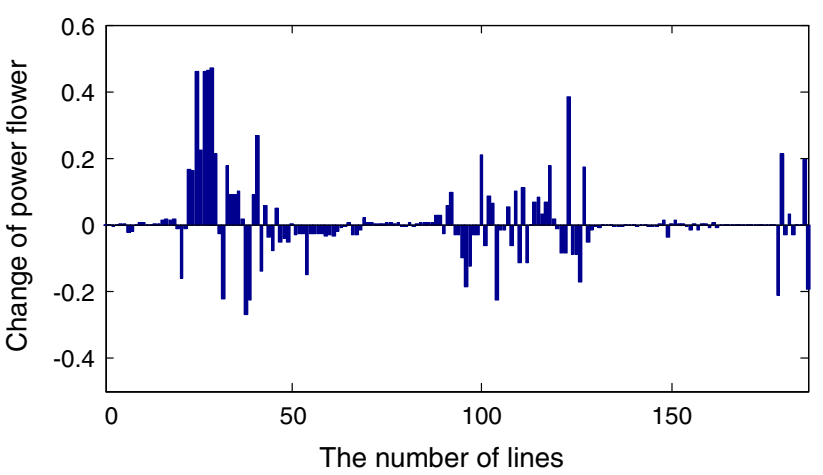

Fig. 7 Power flow changes before and after installation of FACTS devices

rates of lines are in negative growth, indicating that load rates of lines decreased. Fig. 8 shows active power of lines change before and after installation of FACTS devices. The section above zero level shows that active power flow of lines are in positive growth, which means that active power of lines increased. The section below zero level shows that active power flow of lines are in negative growth, which means that active power of lines decreased. By comparing the changes of Fig. 6 and Fig. 7, we know that the active power of line may be negative (positive) before installation of FACTS devices, and the active power of line is likely to become positive (negative) after installation of FACTS devices, which leads to the increase or decrease of the load rate of line.

Tables and figures show that the locations based on power flow entropy have obvious effects on power flow of distribution and load rates to achieve power flow optimization in power systems. Under the objective of power flow entropy, the coordination and optimization of multiFACTS decreases the value of power flow entropy to lower the probability of cascading failures and large blackouts due to chain reaction in power grid and improve the security of the system. Meanwhile, it controls the overload lines, improves the low load of transmission power lines, and reduces the active power generations and the losses of power system.

\section{Conclusions}

In this paper, the value of power flow entropy is minimized as an objective optimization function, subject to the power system limits and economic limits. The modified GSO is effectively and successfully implemented to determine optimal allocation of multi-type of FACTS devices. Based on the case study, the following conclusions are:

1) Only the reasonable locations, number and capacities of FACTS can make the distribution of power flow equilibrium. Otherwise they would make the distribution of power flow more uneven and endanger the system security.

2) In this paper, the proposed method can be relatively accurate for allocation of FACTS devices and advantageous to the distribution of power flow.

3) By changing the value of power flow entropy, we can intuitively learn that TCSC has more ability than SVC for power flow regulation.

Acknowledgment This work was funded by National Science and Technology Support Program of China (2010BAE00816).

Open Access This article is distributed under the terms of the Creative Commons Attribution License which permits any use, distribution, and reproduction in any medium, provided the original author(s) and the source are credited. 


\section{References}

[1] Hingonni NG (1993) Flexible AC transmission. IEEE Spectr 30(4):40-45

[2] Billinton R, Cui Y (2002) Reliability evaluation of composite electric power systems incorporating facts, vol. 1. In: Proceedings of the 2002 IEEE Canadian conference on electrical and computer engineering (CCECE'02), Winnipeg, Canada, 12-15 May 2002

[3] Sinha SK, Patel RN, Prasad R (2011) Applications of FACTS devices with fuzzy controller for oscillation damping in AGC. In: Proceedings of the 2011 international conference on recent advancements in electrical, electronics and control engineering (ICONRAEeCE), Sivakasi, India, 15-17 Dec 2011, pp 314-318

[4] Saravanan M, Slochanal SMR, Venkatesh P et al (2005) Applications of PSO technique for optimal location of facts devices considering system loadability and cost of installation, vol. 2. In: Proceedings of the 7th international power engineering conference (IPEC'05), Singapore, 29 Nov-2 Dec 2005, pp 716-721

[5] Jordeh AR, Jasni J (2012) Approaches for FACTS optimization problem in power systems. In: Proceedings of the 2012 IEEE power engineering and optimization conference (PEDCO'12), Melaka, Malaysia, 6-7 June 2012, pp 355-360

[6] Zhang LZ, Zhao DM (2006) Optimal placement of FACTS for total transfer capability enhancement. Power Syst Technol 30(10):58-62 (in Chinese)

[7] Marouani I, Guesmi T, Abdallah HH et al (2009) Application of a multiobjective evolutionary algorithm for optimal location and parameters of FACTS devices considering the real power loss in transmission lines and voltage deviation buses. In: Proceedings of the 6th international multi-conference on systems, signals and devices (SSD’09), Djerba, 23-26 Mar 2009

[8] Biansoongnern S,Chusanapiputt S, Phoomvuthisarn S (2006) Optimal SVC and TCSC placement for minimization of transmission losses. In: Proceedings of the international conference on power system technology (PowerCon'06), Chongqing, China, 22-26 Oct 2006

[9] Jafarzadeh J, Haq MT, Mahaei SM et al (2011) Optimal placement of FACTS devices based on network security, vol. 3 . In: Proceedings of the 3rd international conference on computer research and development (ICCRD'11), Shanghai, China, 11-13 Mar 2011, pp 345-349

[10] Marouani I, Guesmi T, Hadj Abdallah H et al (2011) Optimal location of multi type FACTS devices for multiple contingencies using genetic algorithms. In: Proceedings of the 8th international multi-conference on systems, signals and devices (SSD'11), Sousse, 22-25 Mar 2011

[11] Huang GM, Li Y (2003) Composite power system reliability evaluation for systems with SVC and TCPAR, vol. 2. In: Proceedings of the 2003 power engineering society general meeting, Toronto, Canada, 13-17 July 2003

[12] Lu Z, Li MS, Tang WJ et al (2007) Optimal location of FACTS devices by a bacterial swarming algorithm for reactive power planning In: Proceedings of the IEEE congress on evolutionary computation (CEC'07), Singapore, 25-28 Sept 2007, pp 2344-2349

[13] Parizad A, Khazal A, Kalantar M (2009) Application of HSA and GA in optimal placement of FACTS devices considering voltage stability and losses. In: Proceedings of the international conference on electric power and energy conversion systems (EPECS'09), Sharjah, 10-12 Nov 2009, pp 1-7

[14] Rashed GI, Shaheen HI, Cheng SJ (2007) Optimal location and parameter settings of multiple TCSCs for increasing power system loadability based on GA and PSO techniques. In:
Proceedings of the 3rd international conference on natural computation, Haikou, China, 24-27 Aug 2007, pp 335-344

[15] Gerbex S, Cherkaoui R, Germond A (2001) Optimal location of multi-type FACTS devices in a power system by means of genetic algorithms. IEEE Trans Power Syst 16(3):537-544

[16] Ongsaku W, Jirapong P (2005) Optimal allocation of FACTS devices to enhance total transfer capability using evolutionary programming, vol. 5. In: Proceedings of the IEEE international symposium on circuits and systems (ISCAS'05), 23-26 May 2005, pp 4175-4178

[17] Bao ZJ, Cao YJ, Wang GZ et al (2009) Analysis of cascading failure in electric grid based on power flow entropy. Phys Lett A 373(34):3032-3040

[18] Zhao L, Park K, Lai YC (2004) Attack vulnerability of scalefree networks due to cascading breakdown. Phys Rev E 70(3): 101136452

[19] Sun X, Lü YC, Gao J et al (2009) Power grid economy and security coordination lean method. Power Syst Technol 33(11):12-17 (in Chinese)

[20] Yi J, Zhou XX, Xiao YN (2007) Determining the self-organized criticality state of power systems by the cascading failures searching method. Proc CSEE 27(25):1-5 (in Chinese)

[21] Wang XF, Chen GR (2002) Synchronization in scale-free dynamical networks: robustness and fragility. IEEE Trans Circuits Syst-I: Fund Theory Appl 49(1):54-62

[22] Yan P, Sekar A (2005) Steady-state analysis of power system having multiple FACTS devices using line-flow-based equations. IEE P-Gener Transm Distrib 152(1):31-39

[23] Oudalov A, Cherkaoui R, Germond AJ et al (2003) Coordinated power flow control by multiple FACTS devices, vol. 3. In: Proceedings of the 2003 IEEE Bologna power tech conference, Bologna, Italy, 23-26 June 2003

[24] Singh SN, David AK (2000) Placement of FACTS devices in open power market, vol. 1. In: Proceedings of the international conference on advances in power system control, operation and management (APSCOM'00), Hong Kong, China, 30 Oct-1 Nov 2000, pp 173-177

[25] Jumaat SA, Musirin I, Othman MM et al (2012) Optimal placement and sizing of multiple FACTS devices installation. In: Proceedings of the IEEE international conference on power and energy, Kota Kinabalu Sabath, Malaysia, 2-5 Dec 2012, pp 145-150

[26] Cai LJ, Erlich I, Stamtsis G (2004) Optimal choice and allocation of FACTS devices in deregulated electricity market using genetic algorithms, vol. 1. In: Proceedings of the 2004 IEEE PES power systems conference and exposition (PSCE'04), New York, USA, 10-13 Oct 2004, pp 201-207

[27] Habur K, O'Leary D (2004) FACTS-flexible AC transmission systems: for cost effective and reliable transmission of electrical energy. Siemens

[28] He S, Wu QH, Saunders JR (2009) Group search optimizer: an optimization algorithm inspired by animal searching behavior. IEEE Trans Evol Comput 13(5):973-990

[29] He S, Li XL (2008) Application of a group search optimization based artificial neural network to machine condition monitoring. In: Proceedings of the 2008 IEEE international conference on emerging technologies and factory automation (ETFA'08), Hamburg, Germany, 15-18 Sept 2008, pp 1260-1266

[30] Wu QH, Lu Z, Li MS et al (2008) Optimal placement of FACTS devices by a group search optimizer with multiple producer. In: Proceedings of the IEEE congress on evolutionary computation (CEC'08), Hong Kong, China, 1-6 June 2008, pp 1033-1039

[31] Ott E (2002) Chaos in dynamical systems. Cambridge University Press, Cambridge

[32] One-line diagram of IEEE 118-bus test system. IIT Power Group, 2003 
[33] Esmin AAA, Lambert-Torres G (2012) Application of particle swarm optimization to optimal power system. Int $\mathbf{J}$ Innov Comput Inf Control 8(3A):1705-1716

Canbing LI received B.Eng and $\mathrm{PhD}$ degree in Electrical Engineering from Tsinghua University in 2001 and 2006 respectively. Currently, he is an associate professor of Hunan University. His research interests include smart grid and energy sustainability.

Liwu XIAO received the B.Eng degrees from Shandong Agricultural University in 2012. Now he is pursuing his master degree in Hunan University. His research interest is intelligent algorithm and its applications to power system optimal operation.

Yijia CAO received $\mathrm{PhD}$ degree from Huazhong University of Science and Technology in 1994. He became a special-term professor of the "Cheung Kong Scholars Program" in Zhejiang University in 2001. Since March 2008, he has been serving as Vice President of Hunan University. His research interests include power system cascading failure, power system information technology, computational intelligence and its applications to smart grid optimal operation.
Qianlong ZHU received the B.Eng and M. Eng degree from Hunan University in 2011 and 2013 respectively. His research interests include intelligent algorithm and its applications to power system optimal operation.

Baling FANG received M.B.A. in business administration from Jilin University in 2011. Now, he is pursuing his $\mathrm{PhD}$ degree in Hunan University. His research interests in power system, cloud computing and intelligent computing.

Yi TAN received the B.Eng degrees from South China University of Technology in 2009. Now, he is pursuing his $\mathrm{PhD}$ degree in Hunan University. His research interests include computational intelligence and its applications to smart grid optimal operation.

Long ZENG received the B. Eng and M. Eng degree from Changsha University of Science \& Technology, Changsha, China in 2009 and 2013 respectively. Now, he is pursuing $\mathrm{PhD}$ degree in Hunan University. His research interests include power system dispatching. 